\title{
Prevalence of Plasmodium falciparum anti-malarial resistance-associated polymorphisms in pfcrt, pfmdr1 and pfnhe 1 in Muheza, Tanzania, prior to introduction of artemisinin combination therapy
}

Nahla B Gadalla ${ }^{1}$, Gloria Tavera ${ }^{1,4}$, Jianbing Mu', Edward R Kabyemela², Michael Fried ${ }^{3}$, Patrick E Duffy ${ }^{3}$, Juliana M Sá and Thomas E Wellems ${ }^{1 *}$

\begin{abstract}
Background: A report of the chloroquine and amodiaquine resistance pfcrt-SVMNT haplotype in Tanzania raises concern about high-level resistance to the artesunate-amodiaquine combination treatment widely employed in Africa. Mutations in the pfmdr 1 multi-drug resistance gene may also be associated with resistance, and a highly polymorphic microsatellite (ms-4760) of the pfnhe1 gene involved in quinine susceptibility has not been surveyed in Tanzania.

Methods: A total of 234 samples collected between 2003 - 2006 from an observational birth cohort of young children in Muheza, Tanzania were analysed. In these children, 141 cases of $P$. falciparum infections were treated with $\mathrm{AQ}$ and 93 episodes were treated with QN. Haplotypes of pfcrt and pfmdr 1 were determined by a Taqman assay, and ms-4760 repeats in pfnhe1 were assessed by nested PCR amplification and direct sequencing. Parasite population diversity was evaluated using microsatellite markers on five different chromosomes.
\end{abstract}

Results: The pfcrt-CVIET haplotype was present alone in 93.6\% (219/234) of the samples over the study period; the wild-type chloroquine- and amodiaquine-sensitive haplotype pfcrt-CVMNK was present in 4.3\% (10/234) of the samples; and both haplotypes were present in $2.1 \%$ (5/234) of the samples. No significant change in wild-type pfcrt-CVMNK prevalence was evident over the 4-year period of the study. The pfcrt-SVMNT haplotype associated with high-level amodiaquine resistance was not detected in this study. The pfmdr 1 locus was genotyped in 178 of these samples. The pfmdr1-YYNY haplotype predominated in 67.4\% (120/178) of infections and was significantly associated with the pfcrt-CVIET haplotype. All samples carried the wild-type pfmdr1-N1042 codon. The ms-4760 repeat on pfnhe1 locus displayed 12 distinct haplotypes with ms-4760-1 predominating in the population. Analysis of these haplotypes showed no association of a particular haplotype with quinine treatment outcome.

Conclusion: The pfcrt-CVIET chloroquine resistance haplotype dominated in the collection of $P$. falciparum samples from Muheza. The pfcrt-SVMNT haplotype, which threatens the efficacy of amodiaquine and was reported in the same time period from Korogwe, Tanzania, $40 \mathrm{Km}$ from Muheza, was not detected. Relative low prevalence of pfcrt-SVMNT in Africa may result from genetic or other factors rendering $P$. falciparum less supportive of this haplotype than in South America or other regions.

Trial registration: Trial Protocol Number: 08-I-N064.

Keywords: Malaria, Drug resistance, Chloroquine, Amodiaquine, Monodesethylamodiaquine, Quinine

\footnotetext{
* Correspondence: twellems@niaid.nih.gov

'Laboratory of Malaria and Vector Research, National Institute of Allergy and Infectious Diseases, NIH, Rockville, MD, USA

Full list of author information is available at the end of the article
} 


\section{Background}

Parasite resistance to anti-malarials including artemisinins $[1,2]$ poses a great challenge to Plasmodium falciparum malaria control efforts. Monitoring parasite haplotypes that predict susceptibility to major anti-malarials can guide treatment policies. Chloroquine (CQ) and amodiaquine (AQ) resistance are linked to mutations in the $P$. falciparum CQ resistance transporter gene pfcrt [3-5]. Two major haplotypes associated with these forms of drug resistance are $p f c r t$-CVIET in Africa and regions of Asia, and $p f c r t$-SVMNT in regions of South America, Asia and The Pacific. The pfcrt-CVIET haplotype swept the African continent in the wake of widespread CQ use [6].

Evidence suggests that the prevalence of the pfcrtCVIET haplotype has subsided with repopulation of CQsensitive pfcrt-CVMNK parasites in several regions where sulphadoxine-pyrimethamine (SP) or artemisinin-based combination therapy (ACT) replaced CQ as drugs recommended for the treatment of $P$. falciparum malaria [7-20]. This phenomenon has not been observed in South America where, despite removal of $\mathrm{CQ}$ and $\mathrm{AQ}$, mutant pfcrtSVMNT parasites remain highly prevalent [21]. A possible explanation for the faster declines of $p f c r t$-CVIET prevalence in Africa and Southeast Asia is that pfcrt-CVIET is less fit than $p f c r t-S V M N T$ relative to wild-type pfcrtCVMNK in the absence of drug pressure [22]. However, much remains to be understood about parasite fitness and drug resistance [23].

Polymorphisms in another transporter gene ( $p f m d r 1)$ encoding P-glycoprotein homologue 1 (PfPgH-1, also known as PfMDR1) have also been associated with $P$. falciparum responses to AQ and its major metabolite, monodesethylamodiaquine (MDAQ), as well as to other drugs including artemisinin derivatives [24-26]. In vitro drug responses from Nigerian isolates suggest that the pfindr1-86Y codon polymorphism may be associated with decreased susceptibility to AQ [27]. This same polymorphism together with pfmdr1-1246Y has been associated with AQ treatment failures [28-30]. Other studies found an increased frequency of the pfmdr1-YYY triplet (codon positions 86, 184, 1246) in recrudescent and recurrent infections after treatment with AQ [30-33] or artesunate-amodiaquine (AS/AQ) [34].

Quinine $(\mathrm{QN})$ remains an efficacious alternative treatment for malaria, including severe malaria and malaria in pregnancy. QN resistance is not widespread in malaria endemic areas possibly due to the multigenic nature of its resistance mechanism. Polymorphisms in both pfcrt $[4,35]$ and pfmdr1 $[25,36]$ have been associated with QN susceptibility in vitro. The $P$. falciparum homologue sodium-hydrogen ion exchanger gene (pfnhe1) located in chromosome 13 has been linked to moderate QN resistance: polymorphisms in one of three microsatellite regions of $p f n h e 1$, namely two or more repeats of DNNND in ms-4760, were associated with a higher $\mathrm{QN} \mathrm{IC}_{50}$ in the progeny of a genetic cross $(\mathrm{HB} 3 \times \mathrm{Dd} 2)$, in cultureadapted clinical isolates and in laboratory lines [37]. Data from patient isolates tested in vitro for their QN response showed an association of two or more DNNND of the pfnhe1-ms-4760 with higher $\mathrm{IC}_{50}$ values in some [38-41] but not all studies [42-44]. In a review of the genetic markers of QN resistance, Okombo and colleagues have grouped the ms-4760 into blocks of particular repeats (block I to VI) [45]. A recent worldwide analysis of ms4760 sequences identified a high diversity of haplotypes as well as an apparent absence of some of these haplotypes from endemic regions of Africa [46].

Reports of the $p f c r t-S_{\text {agt }}$ VMNT (AGT codon for serine) haplotype in Tanzania [47] and Cameroon [48], in addition to the pfcrt- $\mathrm{S}_{\mathrm{tct}} \mathrm{VMNT}$ (TCT codon for serine) haplotype in Angola [49], raise concern whether AQ treatment failures may increase, particularly if the $P$. falciparum parasites also carry pfmdr1 N86 and 1042D codons that, together with $p f c r t$-SVMNT, are associated with the high levels of AQ resistance found in South America [5]. Spread of the $p f c r t-S V M N T$ and $p f m d r 1$ N86/1042D combination would pose a major threat to AS/AQ combination currently used in 25 African countries for uncomplicated malaria [50]. In light of these reports, a survey for the presence of pfcrt-SVMNT and/or pfmdr1 N86/1042D in samples collected from children treated for malaria in Muheza, located approximately 40 kilometres west of Korogwe District where the observation of pfcrtSVMNT was reported [47], was undertaken in this study. In addition, the diversity of pfnhe1 ms-4760 in children treated with QN in this holoendemic region of Tanzania was investigated.

\section{Methods}

\section{Clinical samples}

Samples analysed in this study had been collected from children aged 3 - 33 months as part of the MotherOffspring Malaria Study performed in Muheza, northeastern Tanzania, between 2003 and 2006 (Figure 1). The details of this observational birth cohort study were approved by Tanzanian and US ethical review boards, and signed informed consent was obtained from all mothers for children to participate in the study, as previously reported $[51,52]$. Confirmation of infection with $P$. falciparum was performed by microscopic examination of blood smears. Blood samples from children who presented with symptoms consistent with clinical malaria (headache, nausea, vomiting, temperature $\geq 37.5^{\circ} \mathrm{C}$ ) were stored on filter paper (FP). Parasite levels in the blood were reported as the number of parasites (pa) per 200 white blood cells ( $\mathrm{pa} / 200 \mathrm{WBC})$, and parasitaemia $(\mathrm{pa} / \mu \mathrm{l})$ estimates were calculated by assuming an average of 8000 WBCs $/ \mu \mathrm{l}$. Standard treatment for children with less than 


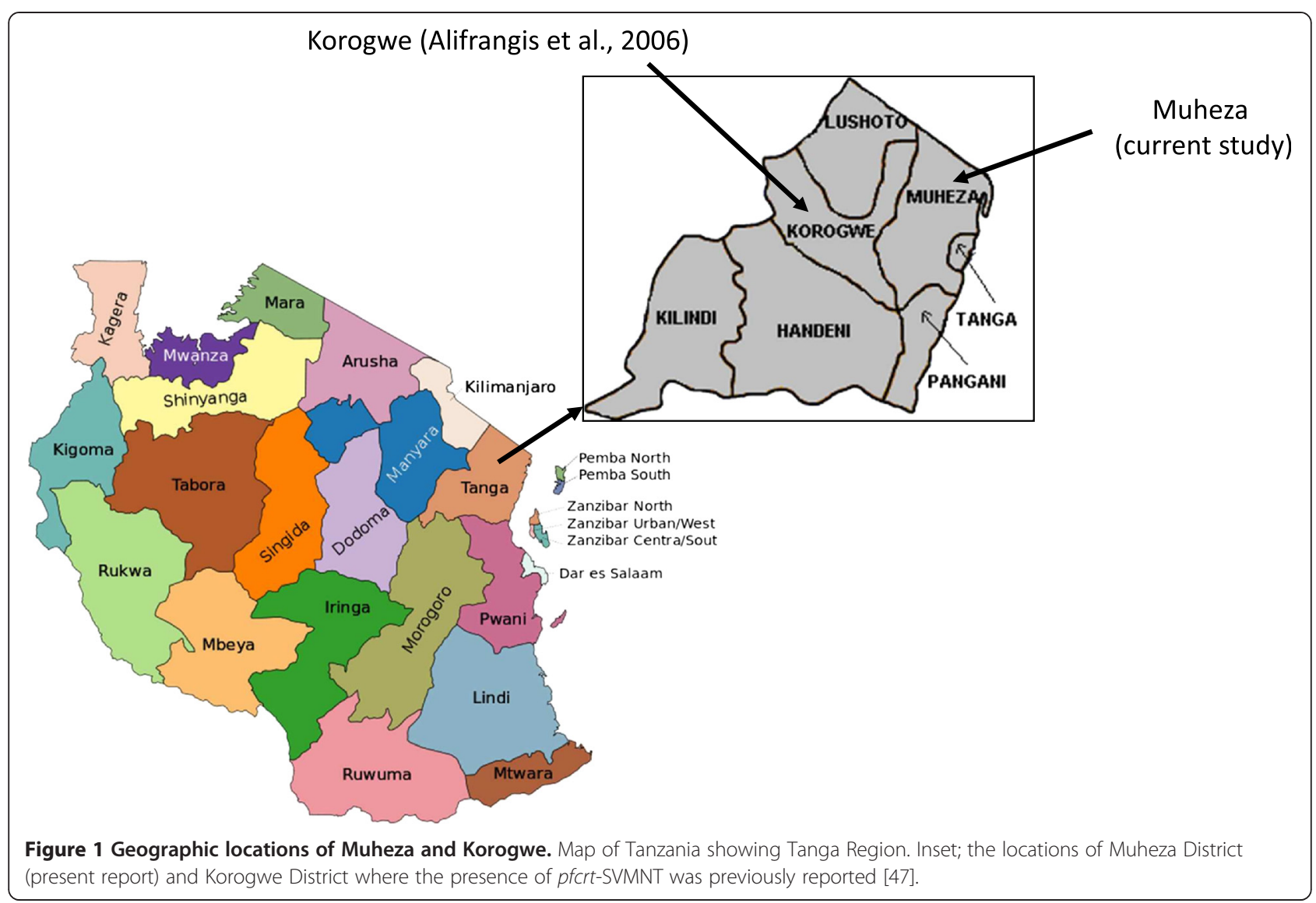

2,500 pa/200 WBC was a 3-day 25-mg/kg AQ regimen $(10,10,5 \mathrm{mg} / \mathrm{kg} /$ day). Children who failed AQ treatment or whose parasitaemia was above $2,500 \mathrm{pa} / 200 \mathrm{WBC}$ were treated with QN at $10 \mathrm{mg} / \mathrm{kg}$ every 8 hours for 7 days [51]. Children unable to take the drugs orally were treated with intravenous QN twice daily until the child was able to take QN orally and complete the 7-day regimen.

\section{DNA extraction and genotyping}

An area of approximately $7 \mathrm{~mm}^{2}$ from FP containing dry blood was cut with a pair of scissors that were decontaminated with $70 \%$ ethanol between samples. The FP was soaked overnight in $20 \%$ Chelex $^{\oplus}$. The supernatant was removed to a clean tube and the FP was washed twice in $1 \mathrm{ml}$ 1X PBS. DNA was extracted using the QIAamp DNA mini kit (Qiagen Inc., USA) per the manufacturer's recommendations and eluted in $50 \mu \mathrm{l}$ of $\mathrm{AE}$ elution buffer. Samples from a non-template control (NTC) obtained by spotting parasite negative $\mathrm{AB}+$ human blood on FP and from a piece of FP not containing any blood were included in each extraction batch (30 samples) to assure absence of contamination.

To assess the pfcrt haplotypes (CVMNK, CVIET, or $\mathrm{S}_{\text {agt }}$ VMNT codons at positions 72-76), a Taqman assay was performed in a $25 \mu \mathrm{l}$ volume containing $12.5 \mu \mathrm{l}$ of
Rotor-Gene Probe PCR Kit (Qiagen Inc., USA), $0.3 \mu \mathrm{M}$ of each primer (PfCRT-D1 and PfCRTD2), $0.1 \mu \mathrm{M}$ of each probe (crt76-CVMNK, crt76-CVIET, crt76-S agt VMNT) and $5 \mu \mathrm{l}$ of sample DNA as previously described [53]. In addition, a probe that detects the $S_{\text {tct }}$ VMNT haplotype (crt76-S tct $_{\text {VMNT 5' }}$ - TCT GTA ATG AAT ACA ATT TTT GCT AA-3') was designed for this study.

Pfmdr1 haplotypes (codons 86, 184, 1042, and 1246) were determined by a Taqman assay employing the primers and probes listed in Table 1. Multiplexed reactions were run for each pair of codons in a single tube in a Rotor Gene 3000 (Qiagen Inc., USA) with four detection channels. The first tube contained primers and probes specific for codons 86 and 1246 and the second tube contained primers and probes specific for codons 184 and 1042. The $25 \mu \mathrm{l}$ reaction contained $12.5 \mu \mathrm{l}$ of Rotor-Gene Multiplex PCR Kit mixture (Qiagen Inc., USA), final concentrations of $0.3 \mu \mathrm{M}$ of each primer, 0.1 $\mu \mathrm{M}$ of each probe and $5 \mu \mathrm{l}$ of DNA. For detection of codons 86 and 1246, samples were subjected to an initial heating step of $95^{\circ} \mathrm{C}$ for $5 \mathrm{~min}$ followed by 40 cycles of $95^{\circ} \mathrm{C}$ for $15 \mathrm{~s}$ and $52^{\circ} \mathrm{C}$ for $30 \mathrm{~s}$. For detection of codons 184 and 1042 , reactions were subjected to an initial heating step of $95^{\circ} \mathrm{C}$ for $5 \mathrm{~min}$ followed by 40 cycles of $95^{\circ} \mathrm{C}$ $15 \mathrm{~s}$ and $50^{\circ} \mathrm{C}$ for $30 \mathrm{~s}$. At least two DNA positive 
Table 1 Primers and probes sequences used to amplify and detect SNPs in pfmdr1

\begin{tabular}{|c|c|c|}
\hline Position & Primer/Probe & Sequence $5^{\prime} \rightarrow 3^{\prime}$ \\
\hline \multirow{4}{*}{86} & Pfmdr1-86F & CCT TाT TाT ATA TCT GTG TIT GGT G \\
\hline & Pfmdr1-86R & TTA TTA TCA TGA AAT TGT CCA TCT TG \\
\hline & Pfmdr1-86N & ROX-AAA GAA CAT GAA TTT AGG TGA TGA-BHQ-2a \\
\hline & Pfmdr1-86Y & Cy5- AAA GAA CAT GTA TाT AGG TGA TGA-BHQ-2a \\
\hline \multirow{4}{*}{184} & Pfmdr1-184F & TTA TAA CAA TाT TTA CAT ATG CCA GTT CC \\
\hline & Pfmdr1-184R & TCT TAT TAC ATA TGA CAC CAC AAA C \\
\hline & Pfmdr1-184Y & JOE-TTA GGT TTA TAT ATT TGG TCA TTAAT-BHQ-1a \\
\hline & Pfmdr1-184F & FAM -TTA GGT TTA TTT ATT TGG TCA TTAAT-BHQ-1a \\
\hline \multirow{4}{*}{1042} & Pfmdr1-1042F & GAA GAA TTA TTG TAA ATG CAG C \\
\hline & Pfmdr1-1042R & CCT TाT AAG GAC ATT AAT TाT C \\
\hline & Pfmdr1-1042N & ROX-ATT ATT TAT TAA TAG TIT TGC-BHQ2a \\
\hline & Pfmdr1-1042D & Cy5-ATT ATT TAT TGA TAG TाT TGC-BHQ2a \\
\hline \multirow{4}{*}{1246} & Pfmdr1-1246F & TGC AGA AGA TTAT ACT GTA TIT AAT A \\
\hline & Pfmdr1-1246R & GCA AAC TTA CTA ACA CGT TTA ACA \\
\hline & Pfmdr1-1246D & JOE- TAT AAC TTA AGA GAT CTT AGA AAC-BHQ-1a \\
\hline & Pfmdr1-1246Y & FAM-TAT AAC TTA AGA TAT CTT AGA AAC- BHQ-1a \\
\hline
\end{tabular}

Mutant bases of the Pfmdr1-86Y, Pfmdr1-184F, Pfmdr1-1042D and Pfmdr1-1246Y primers are shown in bold font. ROX, Cy5, JOE, FAM indicate fluorescent reporter dyes; BHQ-1a and BHQ-2a denote Black Hole Quencher Dyes 1a and 2a, respectively.

controls representing the described codon polymorphisms of P. falciparum clones 3D7, Dd2, HB3 and 7G8 were included in each experiment. Reactions with NTC and FP extractions (described above) and with deionized water substituted for template DNA were employed as negative controls in each experiment. Direct sequencing of the Taqman assay products from 45 samples was also performed to verify the polymorphisms in pfmdr1.

A 350 bp region of the pfuhe1 gene was amplified in 94 of the 99 QN treated samples using nested PCR, as previously described [41]. PCR products were purified by EXOSAP-it (USB, Ohio, USA) and bidirectionally sequenced.

Diversity assessment of 100 randomly selected samples was performed with six polymorphic microsatellite markers present in distinct chromosomes; polya, TA81, TA1, TA60 and TAA87 [54,55], and 299812 [56]. The reverse primer for each marker was fluorescently labeled and PCR products were analysed in an ABI 3730XL Genetic Analyzer (Life Technologies, Carlsbad, CA, USA). Microsatellite data were analysed with Gene Mapper software (v3.2). A mixed microsatellite haplotype was assigned when the minor signal was at least $33.3 \%$ of the major signal [57]. Polyclonality was scored if mixed signals were observed from at least two microsatellites.

\section{Results}

Parasitaemia and treatment outcomes of children receiving $A Q$ or $Q N$

Samples available from the Muheza Mother-Offspring Malaria Study included filter paper blood spots from 141 children who presented to the clinic with a new episode of $P$. falciparum parasitaemia, had no history of antimalarial treatment within the preceding 4 weeks, and were prescribed 3 doses of AQ (Figure 2, see Additional file 1). Of these 141 cases that were treated with AQ, 69 developed microscopic parasitaemia again within four weeks, requiring additional clinical care, while no recurrent parasitaemia was observed in 72 cases. Ninety-three samples were available from cases treated with QN. These samples were from seven cases requiring QN within four weeks of AQ treatment and an additional 86 cases of children presenting with parasite densities $\geq 2,500 \mathrm{pa} / 200$ WBC (est. $100,000 \mathrm{pa} / \mu \mathrm{l} ; 70$ cases) or who were unable to take oral AQ therapy (16 cases; Figure 2).

Presenting parasite densities in the samples from all $141+93=234$ AQ- or QN-treated cases ranged from 40 to $1,119,280 \mathrm{pa} / \mu \mathrm{l}$ with a geometric mean of $30,107 \mathrm{pa} / \mu \mathrm{l}$ (average LN $(\mathrm{pa} / \mu \mathrm{l})=10.313)$. Geometric means of presenting parasite densities were $11,196 \mathrm{pa} / \mu \mathrm{l}$ (average LN $(\mathrm{pa} / \mu \mathrm{l})=9.323)$ in the cases treated with AQ and were $134,903$ (average LN $(\mathrm{pa} / \mu \mathrm{l})=11.812)$ in the cases treated with QN (Table 2). Presenting parasite densities ranged from $40-96,800 \mathrm{pa} / \mu \mathrm{l}$ in cases that showed no recurrence after AQ treatment and from 4,480 - 1,119,280 $\mathrm{pa} / \mu \mathrm{l}$ in cases with no recurrence after QN treatment, while parasite densities in cases of recurrent parasitaemia were 120-204,480 pa/ $\mu \mathrm{l}$ after AQ treatment and 1,680 $744,800 \mathrm{pa} / \mu \mathrm{l}$ after QN treatment. By two-tailed t-tests of $\mathrm{LN}(\mathrm{pa} / \mu \mathrm{l})$ average and standard deviation values, there were no significant differences in the geometric mean 


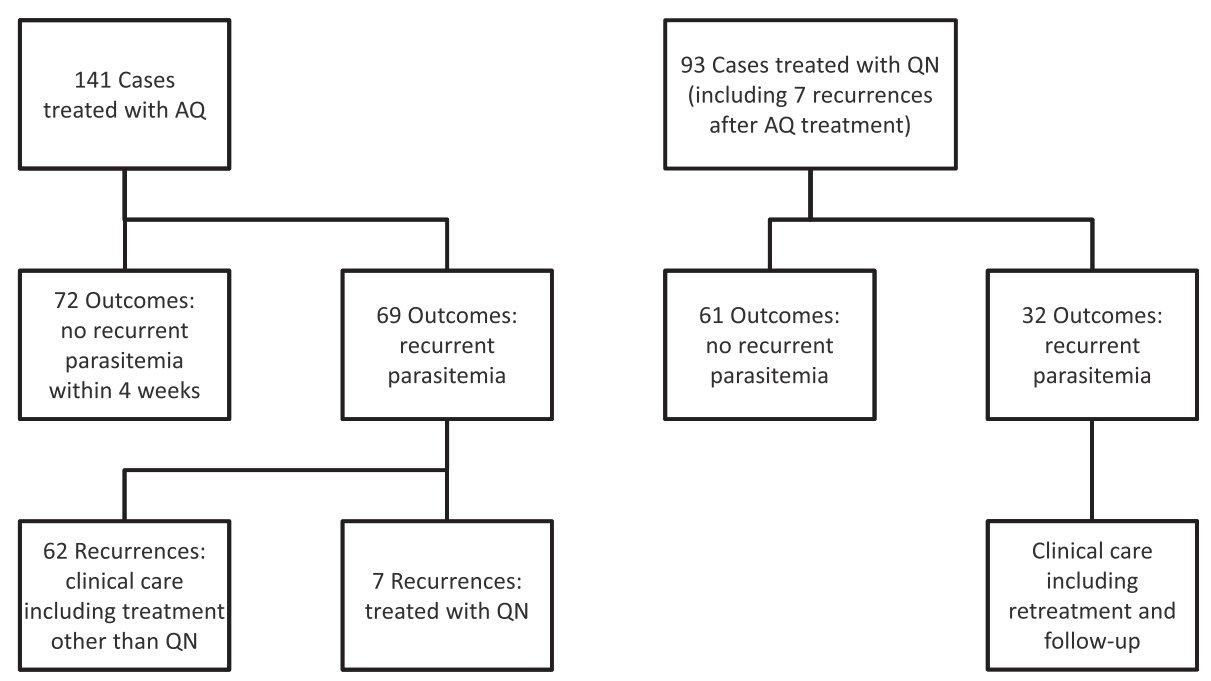

Figure 2 Flowchart of malaria cases, treatments and outcomes in the Muheza Mother-Offspring Malaria Study.

presenting parasitaemia of cases that did or did not develop recurrent parasitaemia after treatment with AQ $(p=0.78)$ or $\mathrm{QN}(p=0.37)$.

\section{pfcrt haplotypes and treatment outcomes in the Muheza cases}

Haplotypes of pfcrt were determined for all 234 cases: 219/234 (93.6\%) of the samples carried pfcrt-CVIET alone, 10/234 (4.3\%) carried pfcrt-CVMNK alone, and 5/ 234. (2.1\%) carried both of these haplotypes (Table 3). The South American pfcrt-SVMNT haplotype was not detected by the Taqman assay or sequencing in any of the samples in this study. By year of collection, the occurrence of pfcrt-CVMNK or mixed pfcrt-CVMNK/ pfcrt-CVIET in the samples was $1 / 32$ in 2003 (3.1\%), 6/ 117 in 2004 (5.1\%), 8/76 in $2005(10.5 \%)$ and $0 / 9$ in 2006 (0.0\%). The different percentages of pfcrt-CVMNK

\section{Table 2 Clinical data of the AQ-treated and QN-treated} groups

\begin{tabular}{lll}
\hline & AQ-treated group & QN-treated group \\
\hline Number of episodes & 141 & 93 \\
Mean $\mathrm{Hb}(\mathrm{g} / \mathrm{dl})$ & 10.5 & 10.6 \\
(range) & $(3.0-18.8)$ & $(4.5-18.3)$ \\
Mean Temperature ${ }^{\circ} \mathrm{C}$ & 37.6 & 38.0 \\
(range) & $(36.0-39.8)$ & $(36.0-40.6)$ \\
Geometric Mean Parasite & 11,196 & 134,903 \\
density $($ pa/ $\mathrm{ll})$ & & $(1,680-1,119,280)$ \\
(range) & $(40-204,480)$ & 86 \\
$\mathrm{Hb} \mathrm{S} \mathrm{AA}$ & 132 & 6 \\
$\mathrm{Hb} \mathrm{S} \mathrm{AS}$ & 7 & 1 \\
$\mathrm{Hb}$ type not determined & 2 & \\
\hline
\end{tabular}

haplotypes in these samples were not statistically significant for a trend between 2003 and 2006 (chi-square test for trend $p=0.27$ ).

Among the cases prescribed AQ and carrying the pfcrtCVIET or mixed pfcrt-CVMNK/pfcrt-CVIET haplotype, only 70/137 (51.1\%) remained clear of recurrent parasitaemia for more than four weeks. Similarly, only $2 / 4$ (50\%) of cases receiving $\mathrm{AQ}$ and carrying pfcrt-CVMNK alone remained clear of recurrent parasitaemia for more than four weeks. QN treatment of infections carrying the pfcrtCVIET or mixed pfcrt-CVMNK/pfcrt-CVIET haplotype cleared $57 / 87(65.5 \%)$ of the parasitaemia for more than four weeks. Similarly, 4/6 (66.7\%) of infections receiving $\mathrm{QN}$ and carrying the pfcrt-CVMNK haplotype alone remained clear of recurrent parasitaemia for more than four weeks.

\section{Distribution of pfmdr1 haplotypes}

From the 234 samples described above, 178 samples were successfully assessed for four pfmdr1 codon polymorphisms: N86 or 86Y; Y184 or 184F; N1042 or 1042D; D1246 or 1246Y (Table 3, Additional file 1). Codons of the $p f m d r 1$-YYNY haplotype either alone or mixed with polymorphisms of other pfmdr1 alleles occurred in 137/ $178(77.0 \%)$ of the samples. Further analysis showed that the codons of pfmdrl-YYNY were present in 135/169 (79.9\%) of the pfcrt-CVIET-containing samples but in only $2 / 9(22.2 \%)$ of samples containing pfcrt-CVMNK alone: this difference was found to be statistically significant (Fishers' exact $p<0.001$ ). In contrast, there was no evident association between pfmdr1-YYND and pfcrt-CVIET (Fisher's Exact $p=0.63$ ). The $p f m d r 1-86 \mathrm{Y}$ polymorphism occurred either alone or mixed with pfmdr1-N86 in 163/ 178 (91.6\%) samples, while pfmdr1-1246Y occurred either alone or mixed with pfmdr1-D1246 in 146/178 (82.0\%) 
Table 3 Distribution of PfCRT and PfMDR1 haplotypes in the study population 2003 - 2006

\begin{tabular}{|c|c|c|c|c|c|}
\hline & \multicolumn{5}{|l|}{ Year } \\
\hline & 2003 & 2004 & 2005 & 2006 & TOTAL \\
\hline \multicolumn{6}{|c|}{ PfCRT amino acid positions $72-76$} \\
\hline CVMNK only & $0.0 \%(0)$ & $3.4 \%(4)$ & $7.9 \%(6)$ & $0 \%(0)$ & $4.3 \%(10)$ \\
\hline CVIET only & $96.9 \%(31)$ & $94.9 \%(111)$ & $89.5 \%(68)$ & $100 \%(9)$ & $93.6 \%(219)$ \\
\hline CVMNK \& CVIET mixed & $3.1 \%(1)$ & $1.7 \%(2)$ & $2.6 \%(2)$ & $0 \%(0)$ & $2.1 \%(5)$ \\
\hline Number of samples & 32 & 117 & 76 & 9 & 234 \\
\hline \multicolumn{6}{|c|}{ PfMDR1 amino acid positions 86, 184, 1042, 1246} \\
\hline YYNY only & $67.7 \%(12)$ & $67.8 \%(59)$ & $66.2 \%(43)$ & $75 \%(6)$ & $67.4 \%(120)$ \\
\hline YYNY mixed* & $5.6 \%(1)$ & $11.5 \%(10)$ & $9.2 \%(6)$ & $0.0 \%(0)$ & $9.6 \%(17)$ \\
\hline YYND only & $16.7 \%(3)$ & $10.3 \%(9)$ & $13.8 \%(9)$ & $12.5 \%(1)$ & $12.4 \%(22)$ \\
\hline YYND mixed** & $0.0 \%(0)$ & $10.3 \%(9)$ & $7.7 \%(5)$ & $0.0 \%(0)$ & $7.9 \%(14)$ \\
\hline Other haplotypes & $11.1 \%(2)$ & $6.9 \%(6)$ & $9.2 \%(6)$ & $12.5 \%(1)$ & $8.4 \%(15)$ \\
\hline Number of samples & 18 & $87^{* * *}$ & $65^{* * *}$ & 8 & $178^{* * *}$ \\
\hline
\end{tabular}

*Mixed with codons of other haplotypes, including 10 YYND (6 in 2004; 4 in 2005).

**Mixed with codons of other haplotypes, including 10 YYNY (6 in 2004; 4 in 2005).

***Total is corrected for the YYNY + YYND mixed samples that are entered twice, as both YYNY mixed and as YYND mixed samples.

samples. The wild-type pfmdr1-Y184 codon was detected in 174/178 (97.8\%) samples. All 178 isolates carried the wild-type $p f m d r 1-\mathrm{N} 1042$ polymorphism.

Of the 178 samples typed for pfmdr1 polymorphisms, 95 were from children who received AQ treatment. AQ cleared $47 / 95$ (49.5\%) of these infections with no recurrence of parasitaemia for more than four weeks. The pfindr1-86Y polymorphism was detected in 45/47 (95.7\%) of children who remained clear of parasitaemia and in $45 / 48$ (93.8\%) of the children who developed recurrent parasitaemia within four weeks (Fishers' exact $p=1.00$ ). The pfmdr1-1246Y polymorphism was detected in $38 / 47$ (78.7\%) of children who remained clear of parasitaemia and in $40 / 48$ (83.3\%) of the children who developed recurrent parasitaemia within four weeks; this difference was also not significant (Fishers' exact $p=0.79$ ).

Eighty-three of the 178 samples typed for $p f m d r 1$ polymorphisms were from children who received QN treatment. QN cleared 56/83 (67.5\%) of these infections with no recurrence of parasitaemia for more than four weeks. The pfmdr1-86Y polymorphism was present in 49/56 (87.5\%) of children who remained clear of parasitaemia and in $24 / 27$ (88.9\%) of the children who developed recurrent parasitaemia within four weeks (Fishers' exact $p=$ 1.00). The pfmdr1-1246Y polymorphism was present in $46 / 56$ (82.1\%) infections of children who remained clear of parasitaemia and in 22/27 (81.5\%) of the children who developed recurrent parasitaemia within four weeks; this difference was not significant (Fishers' exact $p=1.00$ ).

pfnhe1 ms-4760 repeat haplotypes in cases treated with QN Of the 93 cases treated with QN, a 350 bp region of the pfnhe1 gene containing the ms-4760 repeat region was successfully amplified and sequenced from 79/93 (85\%) sample preparations. Twelve different haplotypes of ms4760 were observed, including two previously unreported haplotypes (ms-4760-102 and ms-4760-103; Figure 3). The most prevalent haplotype was ms-4760-1 (two DNNND repeats in block II), observed in 40/79 (50.6\%) of the samples; the second most frequent haplotype, ms4760-3 (one DNNND repeat in block II) occurred in $17.7 \%(14 / 79)$ of samples. Sequences of the observed haplotypes and the number of samples in which each was detected are presented in Figure 3.

Of the 79 QN-treated cases typed for ms-4760, 30.4\% $(24 / 79)$ had returned with parasitaemia within four weeks of treatment, while $69.6 \%$ (55/79) remained clear of parasitaemia. The presence of two or more repeats of DNNND in ms-4670 was not a predictive factor for the return of parasitaemia within 28 days (Fishers' exact $p=0.77$ ). Likewise, there was no association of two or more $v s$ only one DDNHNDNHNND repeat with return of parasitaemia after the QN treatment (Fishers' exact $p=1.0$ ).

\section{Population diversity}

Four polymorphic microsatellites that reside near antimalarial drug resistance loci (MS-Polya, chromosome 4; MS-TAA81, chromosome 5; MS-299812, chromosome 8; MS-TA60, chromosome 13), and 2 microsatellites of chromosome 6 (MS-TA1 and MS-TAA87) were typed in 100 randomly selected samples. For a given microsatellite, a mixed haplotype was assigned if the fluorescence peak from the minor band was at least 33\% of the signal from the major band.

PCR assays of the Polya, MS-TAA81, MS-299812 and MS-TA60 microsatellites detected 28, 19, 19 and 13 size 


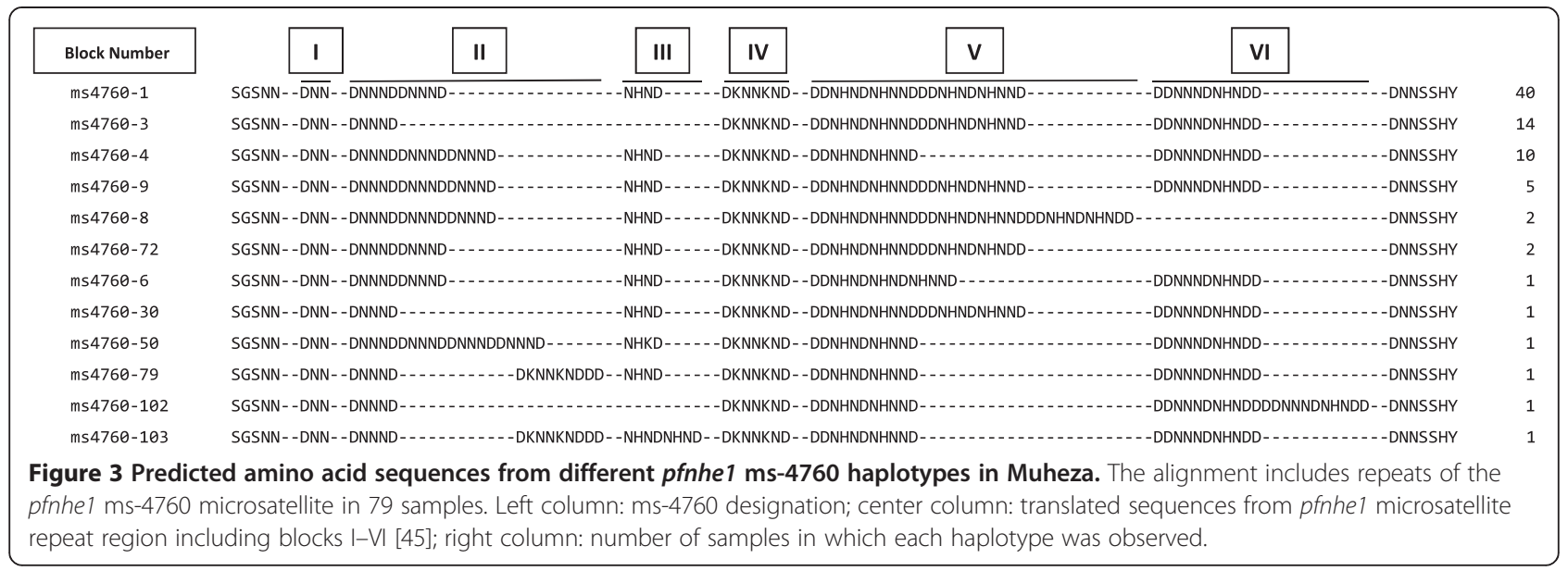

polymorphisms, respectively. For MS-TA60 on chromosome 13, a 70 bp band was obtained from $41.6 \%$ of infections in either a single or mixed haplotype. On chromosome 6, assays of MS-TAA87 detected 38 major and 23 minor size polymorphisms, whereas assays of MS-TA1 detected 23 major and 13 minor polymorphisms. Most infections in the population differed from each other at two or more microsatellites, consistent with a high level of polyclonality and high transmission rates in this area of Tanzania.

\section{Discussion}

Related mechanisms of drug action and resistance are reflected in the structural similarities of $\mathrm{CQ}$ and $\mathrm{AQ}$ as well as the evidence that $p f c r t$ polymorphisms associated with CQ resistance affect AQ efficacy. In a study of $p f c r t$ haplotypes and MDAQ responses in vitro, Warhurst et al. [58] proposed that AQ resistance is associated with the hydrophobicity of $p f c r t$-SVMNT polymorphisms. Amino acid changes encoded by pfmdr 1 that modulate responses of CQ-resistant parasites have also been shown to affect responses to AQ and MDAQ [24]. In the analysis of pfcrt and pfmdr1 of two P. falciparum crosses, Sa et al. [5] found that the highest in vitro $\mathrm{IC}_{50}$ levels for MDAQ occurred in $p f c r t$-CVIET or $p f c r t$-SVMNT progeny carrying either pfmdr1-NFCDY or pfmdr1-NFSDD (codon positions $86,184,1034,1042,1246)$, consistent with the association of the $p f c r t-S V M N T / p f m d r 1-N F C D Y$ combination with AQ failure in South America. These findings together with reports of the pfcrt-SVMNT haplotype in Tanzania, Angola, and Cameroon, raise the concern that if $p f m d r 1$ with the $1042 \mathrm{D}$ change spread into Africa it could promote high-level AQ resistance where this drug is widely deployed as a partner drug in ACT combinations [50].

In the present study, 234 samples collected over a fouryear period from Muheza, Tanzania were analysed for the presence of $p f c r t$ polymorphisms. The mutant $p f c r t$-CVIET (Southeast Asian CQ-resistant form) predominated in the parasite population while the wild type (CQ-sensitive) pfcrt-CVMNK form was present in 1/32 (3.1\%) of samples in $2003,6 / 117(5.1 \%)$ in $2004,8 / 76(10.5 \%)$ in 2005 and $0 / 9(0 \%)$ in 2006; there was no statistically significant trend for $p f c r t$-CVMNK prevalence among these samples over the years. The pfcrt-SVMNT haplotype was not detected in any of the samples analysed in this study. A high level of genetic diversity among all samples was confirmed by six highly polymorphic microsatellites of chromosomes 4, 5, 6 (two markers), 8 and 13. This diversity is an expected outcome in high transmission areas such as Muheza where the entomological inoculation rate has been reported to be 405 infectious bites per year [59].

The pfmdr1-1042D polymorphism that is able to greatly affect MDAQ susceptibility has not been reported in Africa so far; however, three studies on the occurrence of pfcrt-SVMNT in Africa did not provide data for $p f m d r 1$ haplotypes [47-49]. The present study included assessments of 178 Muheza samples for $p f m d r 1$ polymorphisms at codon positions 86, 184, 1042 and 1246. All 178 samples were found to encode only pfimdr1-N1042 with no evidence for the presence of pfmdr1-1042D. Consistent with previous suggestions that $p f c r t$ and $p f m d r 1$ polymorphisms in Asia and Africa may be maintained by substantially different mechanisms than in South America [60], the $p f m d r 1-1042 \mathrm{D}$ amino acid change may be slow to rise in African P. falciparum populations because of genetic background or other factors that are less supportive for this haplotype than in South America. The impediment posed by these factors remains open to question, however, as laboratory experiments have shown that the N1042D codon change can be crossed into the African background (as demonstrated by results from the 7G8/Brazil $\times$ GB4/ Ghana cross [5]). High prevalence in this study of the major pfmdr1-YYNY haplotype in pfcrt-CVIET-carrying parasites is consistent with previous findings of linkage disequilibrium between $p f c r t-76 \mathrm{~T}$ and $p f m d r 1-86 \mathrm{Y}$ polymorphisms [61-63]. 
The diversity of the $p$ fnhe1-ms-4760 repeat in Muheza is similar to the diversity reported from other regions where this repeat has been studied $[41,45,46]$. The ms4760-2 haplotype was not detected, consistent with the absence of this haplotype from a previous report from East Africa [46]. However, ms-4760-4 was observed in ten samples, ms-4760-72 in two samples and ms-4760-79 in one sample; these forms of ms-4760 were not found in previous work from East Africa [46] or in a recent study from Mali [41]. In addition, two haplotypes (ms-4760-102 and ms-4760-103), which have not been previously reported, were observed. A recent report found an association of three DNNND repeats in ms-4760 and higher $\mathrm{IC}_{50}$ for QN in Western Kenya [64]. The results of the present study did not show this association of three DNNND repeats in cases of returned parasitaemia after QN treatment.

Several possible explanations may account for the frequent recurrence of a parasitaemia within 28 days of treatment and the lack of clear associations to known pfcrt, pfmdr1 and pfnhe1 markers of AQ and QN resistance. These include the high entomological inoculation rates that may have contributed frequent reinfections from emergent liver forms as drug levels wane after treatment. The extent of clearance of moderately-resistant parasites carrying pfcrt-CVIET, pfmdr1-86Y, pfmdr1-1246Y or pfnhe1 haplotypes of $\geq 2$ DNNND repeats may have been affected by the immune status of the children [65]. Finally, the authors note that anti-malarial treatments were not fully supervised and plasma drug levels were not followed as part of the parent study to this sub-study; unless disease severity warranted in-patient treatment, children were released from the clinic with medicine and instructions to the parents to complete the prescribed treatment. Compliance and pharmacokinetics of the drugs may have affected treatment efficacy, as has been reported elsewhere [66-69].

Previous studies have documented the return of the wild-type pfcrt-CVMNK haplotype of CQ-sensitivity in parasite populations where alternative drugs including ACT replaced CQ [7-18]. Tanzania changed its malaria treatment policy from CQ to SP in 2001. Although a statistically significant increase of $p f c r t$-CVMNK in Muheza over the period was not found in this study, the presence of this wild-type form in the region may set the stage for the return of increased prevalence of the pfcrt wild-type, as CQ-sensitive parasites in the absence of selective pressure have been observed elsewhere in Tanzania where CQ therapy has also been removed [11]. Selection of opposite alleles of $p f m d r 1$ by ACT partner drugs, such as AQ and lumefantrine [70], suggest that these drugs could be alternated in order to prolong their efficacy.

Occurrence of the $p f c r t-S_{\text {agt }}$ VMNT haplotype in Africa has been reported at low prevalence in countries other than Tanzania: examples include instances of this haplotype in Ghanaian samples collected in 1996-97 [60] and recently from Cameroon [48]. Disparate findings can be found in reports from Angola in 2007 and 2010, where pfcrt-S $\mathrm{S}_{\mathrm{tct}} \mathrm{VMNT}$ was detected in $58 \%$ of 102 samples from Luanda [49], but there was a complete absence of pfcrt-S $\mathrm{S}_{\mathrm{tct}} \mathrm{VMNT}$ from 430 samples collected in Dande [71]. Considering that Luanda and Dande are approximately $90 \mathrm{~km}$ apart, additional surveys will be needed to establish the potential prevalence of $p f c r t-S_{\text {tct }} V M N T$ in this region.

\section{Conclusions}

Although the pfcrt-SVMNT haplotype associated with amodiaquine resistance has been reported from Korogwe, Tanzania, this study of 234 samples from Muheza, approximately 40 kilometers away and in the same time period, did not detect this haplotype. The pfcrt-CVIET haplotype widely responsible for chloroquine resistance was most prevalent in this study and was significantly associated with the pfmdr1-YYNY haplotype. Low levels of the wild-type $p f c r t$-CVMNK haplotype in Muheza may set the stage for the return of chloroquine sensitive $P$. falciparum populations, as has been reported in other regions where chloroquine use was stopped. Relative absence of pfcrt-SVMNT from Africa may reflect genetic or other factors affecting P. falciparum that are less supportive for this haplotype than in South America and other continental regions.

\section{Additional file}

Additional file 1: Clinical information, treatment outcomes and haplotypes of pfcrt, pfmdr1 and pfnhe1 from children in the Muheza Mother-Offspring Malaria Study.

\section{Competing interests}

The authors declare that they have no competing interests.

\section{Authors' contributions}

NBG designed the pfmdr1 Taqman assays, performed the molecular studies, data analysis and drafted the manuscript. GT carried out the sequencing experiments and alignment. JM participated in microsatellite and sequencing experiments. MF, ERK and PD conceived and designed the clinical study and were responsible for sample collection. JMS conceived and designed the molecular study and revised the manuscript. TEW conceived the molecular study, drafted and revised the manuscript. All authors read and approved the final manuscript.

\section{Acknowledgements}

This work was supported by the Division of Intramural Research (DIR) and the extramural program (grant R01Al52059) of NIAID, NIH. We thank the mothers and their children in Muheza District for participation in the studies, and the clinical and laboratory teams of the MOMS Project for data and sample collections. Dr. Whitney Harrington and Robert Morrison advised on the organization and processing of samples for this analysis.

\section{Author details}

${ }^{1}$ Laboratory of Malaria and Vector Research, National Institute of Allergy and Infectious Diseases, NIH, Rockville, MD, USA. ${ }^{2}$ Muhimbili University of Health 
and Allied Sciences, Dar es Salaam, Tanzania. ${ }^{3}$ Laboratory of Malaria Immunology and Vaccinology, National Institute of Allergy and Infectious Diseases, NIH, Rockville, MD, USA. ${ }^{4}$ Case Western Reserve University School of Medicine, Cleveland, OH 44106, USA.

\section{Received: 28 October 2014 Accepted: 7 March 2015 Published online: 25 March 2015}

\section{References}

1. Noedl H, Se Y, Schaecher K, Smith BL, Socheat D, Fukuda MM. Evidence of artemisinin-resistant malaria in western Cambodia. N Engl J Med. 2008;359:2619-20.

2. Dondorp AM, Nosten F, Yi P, Das D, Phyo AP, Tarning J, et al. Artemisinin resistance in Plasmodium falciparum malaria. N Engl J Med. 2009;361:455-67.

3. Fidock DA, Nomura T, Talley AK, Cooper RA, Dzekunov SM, Ferdig MT, et al. Mutations in the $P$. falciparum digestive vacuole transmembrane protein PfCRT and evidence for their role in chloroquine resistance. Mol Cell. 2000;6:861-71

4. Sidhu AB, Verdier-Pinard D, Fidock DA. Chloroquine resistance in Plasmodium falciparum malaria parasites conferred by pfcrt mutations. Science. 2002;298:210-3.

5. Sa JM, Twu O, Hayton K, Reyes S, Fay MP, Ringwald P, et al. Geographic patterns of Plasmodium falciparum drug resistance distinguished by differential responses to amodiaquine and chloroquine. Proc Natl Acad Sci U S A. 2009;106:18883-9.

6. Wootton JC, Feng X, Ferdig MT, Cooper RA, Mu J, Baruch DI, et al. Genetic diversity and chloroquine selective sweeps in Plasmodium falciparum. Nature. 2002:418:320-3.

7. Kublin JG, Cortese JF, Njunju EM, Mukadam RA, Wirima JJ, Kazembe PN, et al. Reemergence of chloroquine-sensitive Plasmodium falciparum malaria after cessation of chloroquine use in Malawi. J Infect Dis. 2003;187:1870-5.

8. Mita T, Kaneko A, Lum JK, Bwijo B, Takechi N, Zungu IL, et al. Recovery of chloroquine sensitivity and low prevalence of the Plasmodium falciparum chloroquine resistance transporter gene mutation K76T following the discontinuance of chloroquine use in Malawi. Am J Trop Med Hyg. 2003;68:413-5.

9. Wang XH, Mu JB, Li GQ, Chen PQ, Guo XB, Fu LC, et al. Decreased prevalence of the Plasmodium falciparum chloroquine resistance transporter 76 T marker associated with cessation of chloroquine use against $P$. falciparum malaria in Hainan, People's Republic of China. Am J Trop Med Hyg. 2005;72:410-4.

10. Laufer MK, Thesing PC, Eddington ND, Masonga R, Dzinjalamala FK, Takala SL, et al. Return of chloroquine antimalarial efficacy in Malawi. N Engl J Med. 2006;355:1959-66

11. Alifrangis $M$, Lusingu JP, Mmbando $B$, Dalgaard MB, Vestergaard LS, Ishengoma $D$, et al. Five-year surveillance of molecular markers of Plasmodium falciparum antimalarial drug resistance in Korogwe District, Tanzania: accumulation of the $581 \mathrm{G}$ mutation in the $P$. falciparum dihydropteroate synthase gene. Am J Trop Med Hyg. 2009;80:523-7.

12. Mwai L, Ochong E, Abdirahman A, Kiara SM, Ward S, Kokwaro G, et al. Chloroquine resistance before and after its withdrawal in Kenya. Malar J. 2009:8:106.

13. Happi CT, Gbotosho GO, Folarin OA, Sowunmi A, Hudson T, O'Neil M, et al. Selection of Plasmodium falciparum multidrug resistance gene 1 alleles in asexual stages and gametocytes by artemether-lumefantrine in Nigerian children with uncomplicated falciparum malaria. Antimicrob Agents Chemother. 2009;53:888-95.

14. Laufer MK, Takala-Harrison S, Dzinjalamala FK, Stine OC, Taylor TE, Plowe CV. Return of chloroquine-susceptible falciparum malaria in Malawi was a reexpansion of diverse susceptible parasites. J Infect Dis. 2010;202:801-8.

15. Some AF, Sere YY, Dokomajilar C, Zongo I, Rouamba N, Greenhouse B, et al. Selection of known Plasmodium falciparum resistance-mediating polymorphisms by artemether-lumefantrine and amodiaquine-sulfadoxinepyrimethamine but not dihydroartemisinin-piperaquine in Burkina Faso. Antimicrob Agents Chemother. 2010;54:1949-54.

16. Gadalla NB, Abdallah TM, Atwal S, Sutherland CJ, Adam I. Selection of pfdhfr/pfdhps alleles and declining artesunate/sulphadoxine-pyrimethamine efficacy against Plasmodium falciparum eight years after deployment in eastern Sudan. Malar J. 2013;12:255.

17. Thomsen TT, Madsen LB, Hansson HH, Tomas EV, Charlwood D, Bygbjerg IC, et al. Rapid selection of Plasmodium falciparum chloroquine resistance transporter gene and multidrug resistance gene-1 haplotypes associated with past chloroquine and present artemether-lumefantrine use in Inhambane District, southern Mozambique. Am J Trop Med Hyg. 2013;88:536-41.

18. Duah NO, Matrevi SA, de Souza DK, Binnah DD, Tamakloe MM, Opoku VS, et al. Increased pfmdrl gene copy number and the decline in pfcrt and pfmdr 1 resistance alleles in Ghanaian Plasmodium falciparum isolates after the change of anti-malarial drug treatment policy. Malar J. 2013;12:377.

19. Mvumbi DM, Boreux R, Sacheli R, Lelo M, Lengu B, Nani-Tuma S, et al. Assessment of pfcrt 72-76 haplotypes eight years after chloroquine withdrawal in Kinshasa. Democratic Republic of Congo Malar J. 2013;12:459.

20. Frosch AE, Laufer MK, Mathanga DP, Takala-Harrison S, Skarbinski J, Claassen CW et al. Return of widespread chloroquine-sensitive Plasmodium falciparum to Malawi. J Infect Dis. 2014;210:1110-4.

21. Gbotosho GO, Folarin OA, Bustamante C, da Silva LH, Mesquita E, Sowunmi $A$, et al. Different patterns of pfcrt and pfmdr1 polymorphisms in $P$. falciparum isolates from Nigeria and Brazil: the potential role of antimalarial drug selection pressure. Am J Trop Med Hyg. 2012:86:211-3.

22. Sa JM, Twu O. Protecting the malaria drug arsenal: halting the rise and spread of amodiaquine resistance by monitoring the PfCRT SVMNT type. Malar J. 2010;9:374

23. Rosenthal PJ. The interplay between drug resistance and fitness in malaria parasites. Mol Microbiol. 2013;89:1025-38.

24. Duraisingh MT, Drakeley CJ, Muller O, Bailey R, Snounou G, Targett GA, et al. Evidence for selection for the tyrosine-86 allele of the pfmdr 1 gene of Plasmodium falciparum by chloroquine and amodiaquine. Parasitology. 1997;114(Pt 3):205-11.

25. Duraisingh MT, Roper C, Walliker D, Warhurst DC. Increased sensitivity to the antimalarials mefloquine and artemisinin is conferred by mutations in the pfmdr1 gene of Plasmodium falciparum. Mol Microbiol. 2000;36:955-61.

26. Reed MB, Saliba KJ, Caruana SR, Kirk K, Cowman AF. Pgh1 modulates sensitivity and resistance to multiple antimalarials in Plasmodium falciparum. Nature. 2000;403:906-9.

27. Folarin OA, Bustamante C, Gbotosho GO, Sowunmi A, Zalis MG, Oduola AM, et al. In vitro amodiaquine resistance and its association with mutations in pfcrt and pfmdr1 genes of Plasmodium falciparum isolates from Nigeria. Acta Trop. 2011;120:224-30.

28. Happi CT, Gbotosho GO, Folarin OA, Bolaji OM, Sowunmi A, Kyle DE, et al. Association between mutations in Plasmodium falciparum chloroquine resistance transporter and $P$. falciparum multidrug resistance 1 genes and in vivo amodiaquine resistance in $P$. falciparum malaria-infected children in Nigeria. Am J Trop Med Hyg. 2006;75:155-61.

29. Djimde AA, Fofana B, Sagara I, Sidibe B, Toure S, Dembele D, et al. Efficacy, safety, and selection of molecular markers of drug resistance by two ACTs in Mali. Am J Trop Med Hyg. 2008;78:455-61.

30. Danquah I, Coulibaly B, Meissner P, Petruschke I, Muller O, Mockenhaupt FP. Selection of pfmdr1 and pfcrt alleles in amodiaquine treatment failure in north-western Burkina Faso. Acta Trop. 2010;114:63-6.

31. Holmgren G, Hamrin J, Svard J, Martensson A, Gil JP, Bjorkman A. Selection of pfmdr 1 mutations after amodiaquine monotherapy and amodiaquine plus artemisinin combination therapy in East Africa. Infect Genet Evol. 2007;7:562-9.

32. Nsobya SL, Dokomajilar C, Joloba M, Dorsey G, Rosenthal PJ. Resistance-mediating Plasmodium falciparum pfcrt and pfmdr1 alleles after treatment with artesunate-amodiaquine in Uganda. Antimicrob Agents Chemother. 2007;51:3023-5.

33. Humphreys GS, Merinopoulos I, Ahmed J, Whitty CJ, Mutabingwa TK, Sutherland CJ, et al. Amodiaquine and artemether-lumefantrine select distinct alleles of the Plasmodium falciparum mdr1 gene in Tanzanian children treated for uncomplicated malaria. Antimicrob Agents Chemother. 2007:51:991-7.

34. Thwing Jl, Odero CO, Odhiambo FO, Otieno KO, Kariuki S, Ord R, et al. In-vivo efficacy of amodiaquine-artesunate in children with uncomplicated Plasmodium falciparum malaria in western Kenya. Trop Med Int Health. 2009;14:294-300.

35. Cooper RA, Lane KD, Deng B, Mu J, Patel JJ, Wellems TE, et al. Mutations in transmembrane domains 1, 4 and 9 of the Plasmodium falciparum chloroquine resistance transporter alter susceptibility to chloroquine, quinine and quinidine. Mol Microbiol. 2007;63:270-82.

36. Duraisingh MT, Cowman AF. Contribution of the pfmdr1 gene to antimalarial drug-resistance. Acta Trop. 2005;94:181-90. 
37. Ferdig MT, Cooper RA, Mu J, Deng B, Joy DA, Su XZ, et al. Dissecting the loci of low-level quinine resistance in malaria parasites. Mol Microbiol. 2004:52:985-97.

38. Henry M, Briolant S, Zettor A, Pelleau S, Baragatti M, Baret E, et al. Plasmodium falciparum $\mathrm{Na}+/ \mathrm{H}+$ exchanger 1 transporter is involved in reduced susceptibility to quinine. Antimicrob Agents Chemother. 2009;53:1926-30.

39. Okombo J, Kiara SM, Rono J, Mwai L, Pole L, Ohuma E, et al. In vitro activities of quinine and other antimalarials and pfnhe polymorphisms in Plasmodium isolates from Kenya. Antimicrob Agents Chemother. 2010;54:3302-7.

40. Sinou V, le Quang $H$, Pelleau S, Huong VN, Huong NT, le Tai M, et al Polymorphism of Plasmodium falciparum $\mathrm{Na}(+) / \mathrm{H}(+)$ exchanger is indicative of a low in vitro quinine susceptibility in isolates from Viet Nam. Malar J. 2011;10:164.

41. Kone A, Mu J, Maiga H, Beavogui AH, Yattara O, Sagara I, et al. Quinine treatment selects the pfnhe-1 ms4760-1 polymorphism in Malian patients with falciparum malaria. J Infect Dis. 2013;207:520-7.

42. Andriantsoanirina V, Menard D, Rabearimanana S, Hubert V, Bouchier C, Tichit M, et al. Association of microsatellite variations of Plasmodium falciparum $\mathrm{Na}+\mathrm{H}+$ exchanger (Pfnhe-1) gene with reduced in vitro susceptibility to quinine: lack of confirmation in clinical isolates from Africa. Am J Trop Med Hyg. 2010;82:782-7.

43. Baliraine FN, Nsobya SL, Achan J, Tibenderana JK, Talisuna AO, Greenhouse B, et al. Limited ability of Plasmodium falciparum pfcrt, pfmdr1, and pfnhe1 polymorphisms to predict quinine in vitro sensitivity or clinical effectiveness in Uganda. Antimicrob Agents Chemother. 2011;55:615-22

44. Briolant S, Pelleau S, Bogreau H, Hovette P, Zettor A, Castello J, et al. In vitro susceptibility to quinine and microsatellite variations of the Plasmodium falciparum $\mathrm{Na}+/ \mathrm{H}+$ exchanger (Pfnhe-1) gene: the absence of association in clinical isolates from the Republic of Congo. Malar J. 2011;10:37.

45. Okombo J, Ohuma E, Picot S, Nzila A. Update on genetic markers of quinine resistance in Plasmodium falciparum. Mol Biochem Parasitol. 2011;177:77-82.

46. Menard D, Andriantsoanirina V, Khim N, Ratsimbasoa A, Witkowski B, Benedet $\mathrm{C}$, et al. Global analysis of Plasmodium falciparum $\mathrm{Na}(+) / \mathrm{H}(+)$ exchanger (pfnhe-1) allele polymorphism and its usefulness as a marker of in vitro resistance to quinine. Int J Parasitol Drugs Drug Resist. 2013;3:8-19.

47. Alifrangis M, Dalgaard MB, Lusingu JP, Vestergaard LS, Staalsoe $T$, Jensen AT, et al. Occurrence of the Southeast Asian/South American SVMNT haplotype of the chloroquine-resistance transporter gene in Plasmodium falciparum in Tanzania. J Infect Dis. 2006;193:1738-41.

48. Ngassa Mbenda HG, Das A. Occurrence of multiple chloroquine-resistant Pfcrt haplotypes and emergence of the S(agt) VMNT type in Cameroonian Plasmodium falciparum. J Antimicrob Chemother. 2014;69:400-3.

49. Gama BE, Pereira-Carvalho GA, Lutucuta Kosi FJ, de Oliveira NK A, Fortes F, Rosenthal PJ, et al. Plasmodium falciparum isolates from Angola show the StctVMNT haplotype in the pfcrt gene. Malar J. 2010;9:174

50. WHO. World Malaria Report : 2013. Geneva: World Health Organization; 2013.

51. Mutabingwa TK, Bolla MC, Li JL, Domingo GJ, Li X, Fried M, et al. Maternal malaria and gravidity interact to modify infant susceptibility to malaria. PLoS Med. 2005;2:e407.

52. Harrington WE, Mutabingwa TK, Kabyemela E, Fried M, Duffy PE. Intermittent treatment to prevent pregnancy malaria does not confer benefit in an area of widespread drug resistance. Clin Infect Dis. 2011:53:224-30

53. Gadalla NB, Elzaki SE, Mukhtar E, Warhurst DC, El-Sayed B, Sutherland CJ. Dynamics of pfcrt alleles CVMNK and CVIET in chloroquine-treated Sudanese patients infected with Plasmodium falciparum. Malar J. 2010;9:74

54. Anderson TJ, Su XZ, Bockarie M, Lagog M, Day KP. Twelve microsatellite markers for characterization of Plasmodium falciparum from finger-prick blood samples. Parasitology. 1999;119(Pt 2):113-25.

55. Anderson TJ, Nair S, Qin H, Singlam S, Brockman A, Paiphun L, et al. Are transporter genes other than the chloroquine resistance locus ( $p f c r t$ ) and multidrug resistance gene ( $p f m d r$ ) associated with antimalarial drug resistance? Antimicrob Agents Chemother. 2005:49:2180-8.

56. Anderson TJ, Nair S, Nkhoma S, Williams JT, Imwong M, Yi P, et al. High heritability of malaria parasite clearance rate indicates a genetic basis for artemisinin resistance in western Cambodia. J Infect Dis. 2010;201:1326-30.

57. Anderson TJ, Haubold B, Williams JT, Estrada-Franco JG, Richardson L, Mollinedo $\mathrm{R}$, et al. Microsatellite markers reveal a spectrum of population structures in the malaria parasite Plasmodium falciparum. Mol Biol Evol. 2000:17:1467-82.

58. Warhurst DC. Polymorphism in the Plasmodium falciparum chloroquine-resistance transporter protein links verapamil enhancement of chloroquine sensitivity with the clinical efficacy of amodiaquine. Malar J. 2003;2:31.

59. Ellman R, Maxwell C, Finch R, Shayo D. Malaria and anaemia at different altitudes in the Muheza district of Tanzania: childhood morbidity in relation to level of exposure to infection. Ann Trop Med Parasitol. 1998;92:741-53.

60. Mehlotra RK, Mattera G, Bockarie MJ, Maguire JD, Baird JK, Sharma YD, et al. Discordant patterns of genetic variation at two chloroquine resistance loci in worldwide populations of the malaria parasite Plasmodium falciparum. Antimicrob Agents Chemother. 2008:52:2212-22.

61. Duraisingh MT, von Seidlein LV, Jepson A, Jones P, Sambou I, Pinder M, et al. Linkage disequilibrium between two chromosomally distinct loci associated with increased resistance to chloroquine in Plasmodium falciparum. Parasitology. 2000;121(Pt 1):1-7.

62. Adagu IS, Warhurst DC. Plasmodium falciparum: linkage disequilibrium between loci in chromosomes 7 and 5 and chloroquine selective pressure in Northern Nigeria. Parasitology. 2001;123:219-24.

63. Babiker HA, Pringle SJ, Abdel-Muhsin A, Mackinnon M, Hunt P, Walliker D. High-level chloroquine resistance in Sudanese isolates of Plasmodium falciparum is associated with mutations in the chloroquine resistance transporter gene pfcrt and the multidrug resistance gene pfmdr1. J Infect Dis. 2001;183:1535-8

64. Cheruiyot J, Ingasia LA, Omondi AA, Juma DW, Opot BH, Ndegwa JM, et al. Polymorphisms in Pfmdr1, Pfcrt, and Pfnhel genes are associated with reduced in vitro activities of quinine in Plasmodium falciparum isolates from western Kenya. Antimicrob Agents Chemother. 2014;58:3737-43.

65. Djimde AA, Doumbo OK, Traore O, Guindo AB, Kayentao K, Diourte Y, et al. Clearance of drug-resistant parasites as a model for protective immunity in Plasmodium falciparum malaria. Am J Trop Med Hyg. 2003;69:558-63.

66. Djimde A, Plowe CV, Diop S, Dicko A, Wellems TE, Doumbo O. Use of antimalarial drugs in Mali: policy versus reality. Am J Trop Med Hyg. 1998;59:376-9.

67. Amin AA, Hughes DA, Marsh V, Abuya TO, Kokwaro GO, Winstanley PA et al. The difference between effectiveness and efficacy of antimalarial drugs in Kenya. Trop Med Int Health. 2004;9:967-74.

68. Yeung $\mathrm{S}$, White NJ. How do patients use antimalarial drugs? A review of the evidence. Trop Med Int Health. 2005;10:121-38.

69. Souares A, Lalou R, Sene I, Sow D, Le Hesran JY. Factors related to compliance to anti-malarial drug combination: example of amodiaquine/ sulphadoxine-pyrimethamine among children in rural Senegal. Malar J. 2009;8:118.

70. Venkatesan M, Gadalla NB, Stepniewska K, Dahal P, Nsanzabana C, Moriera $\mathrm{C}$, et al. Polymorphisms in Plasmodium falciparum chloroquine resistance transporter and multidrug resistance 1 genes: parasite risk factors that affect treatment outcomes for $P$. falciparum malaria after artemether-lumefantrine and artesunate-amodiaquine. Am J Trop Med Hyg. 2014;91:833-43.

71. Fancony C, Gamboa D, Sebastiao Y, Hallett R, Sutherland C, Sousa-Figueiredo JC, et al. Various pfcrt and pfmdr1 genotypes of Plasmodium falciparum cocirculate with P. malariae, P. ovale spp., and P. vivax in northern Angola. Antimicrob Agents Chemother. 2012;56:5271-7.

\section{Submit your next manuscript to BioMed Central and take full advantage of:}

- Convenient online submission

- Thorough peer review

- No space constraints or color figure charges

- Immediate publication on acceptance

- Inclusion in PubMed, CAS, Scopus and Google Scholar

- Research which is freely available for redistribution 